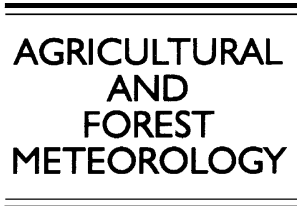

\title{
A practical scheme for correcting multiple scattering effects on optical LAI measurements
}

\author{
Sylvain G. Leblanc ${ }^{\mathrm{a}, *}$, Jing M. Chen ${ }^{\mathrm{b}}$ \\ ${ }^{a}$ Canada Centre for Remote Sensing, 588 Booth Street, 4th Floor, Ottawa, Ont., Canada K1A $0 Y 7$ \\ ${ }^{\mathrm{b}}$ University of Toronto, 100 St. George St., Room 5047, Toronto, Ont., Canada M5S 3G3
}

Received 8 April 2001; received in revised form 10 September 2001; accepted 19 October 2001

\begin{abstract}
Accurate and fast non-destructive measurements of leaf area index (LAI) of plant canopies are essential to environmental applications such as water and carbon cycle modelling. A commonly used technique to acquire LAI in situ is based on measurements of radiation transmittance through the canopy with optical instruments. The LAI-2000, that obtains measurements of effective LAI ( $L_{\mathrm{e}}$ : LAI retrieved assuming random foliage distribution) based on gap fraction at five view angles, is designed to work under diffuse light conditions. The LAI-2000 makes use of blue light to minimise the effect of light scattering in the canopy on LAI measurements. However, actual field LAI measurements are still routinely done under a range of illumination conditions, including direct sunlight. The LAI values measured under conditions of either partial or full direct light are generally smaller than the ones obtained under diffuse conditions. Although this error source is prevailing in many field LAI measurements, hitherto the problem has not been tackled rigorously. To better understand and improve the LAI-2000 measurements taken under non-ideal conditions, measurements were taken in two deciduous and two coniferous forest sites at different times of cloudless days to study how the scattering of the blue light by plant canopies affects LAI measurements. The sites are located in Larose forest near Ottawa, Canada. It is shown through these measurements and modelling with the canopy radiative transfer model Five-scale [Remote Sens. Rev. 19 (2000) 293-305] that the blue light scattering causes underestimation of effective LAI by up to $20 \%$ when measured under direct sunlight. A correction for the scattering effect, as a function of solar zenith angle and the effective LAI measured under the sunlit condition, is found through an empirical fit to the measured data in a limited range as well as model-simulated data in the full possible range possible. It is also found that the LAI-2000 fourth ring ( $47-58^{\circ}$ from zenith) gives a more consistent correction than the other rings and that this ring used alone is also suitable for effective LAI retrieval under diffuse conditions. The correction scheme can reduce the error in effective LAI measurements to within $2 \%$. It is therefore suggested that in field programs with logistic constraints, the LAI-2000 time of operation during sunny days can be extended beyond the diffuse illumination conditions near sunrise and sunset since the influence of the direct sunlight on the LAI measurements can be mostly removed using the correction scheme provided in this study. ( 2001 Published by Elsevier Science B.V.
\end{abstract}

Keywords: Multiple scattering effect; Leaf area index; Larose forest; Plant canopies

\footnotetext{
* Corresponding author. Tel.: +1-613-947-1294;

fax: +1-613-947-1406.

E-mail addresses: sylvain.leblanc@ccrs.nrcan.gc.ca (S.G. Leblanc), chenj@geog.utoronto.ca (J.M. Chen).
}

\section{Introduction}

The leaf area index (LAI) is an important biophysical parameter used in carbon budget models and in climate, weather and ecological studies (Badhwar et al., 1986; Liu et al., 1997). The definition of LAI as one 
half the total leaf area per unit ground surface area is used here instead of the definition based on the projected (one-sided) area (Ross, 1981) because when the foliage angular distribution is random, the usual projection coefficient of 0.5 can still be used for foliage of any shape (Chen and Black, 1992). LAI and its angular and spatial distributions define the interception of solar radiation by plant canopies responsible for carbon absorption and exchange with the atmosphere. LAI measurements are usually done either directly through sampling (destructive sampling or litter collection) and allometric techniques (Gower et al., 1999), or from gap fraction estimates made with optical instruments (Chen et al., 1997). Destructive sampling is time consuming and labour intensive, and litter collection does not allow seasonal variations to be measured. Moreover, the allometric relationships found from these measurements are subject to many uncertainties when scaled from a few sample plants to a whole canopy (Chen et al., 1997). Thus, the optical technique is often the preferred choice because the LAI estimation can be done rapidly with accuracy comparable to destructive sampling (Chen et al., 1997), up to a LAI of six (Gower et al., 1999) at which point the gap fraction can saturate and direct measurement is often the only reliable way of measuring LAI.

There are presently several optical instruments commercially available for measuring LAI. These instruments require specific illumination conditions. The LAI-2000 (Plant Canopy Analyser, LI-COR, Lincoln, NE) makes use of diffuse light and should, in principle, avoid direct sunlight. Therefore, the measurements should be taken on uniform sky conditions found on overcast days, or near sunset or sunrise to avoid the interference of direct sunlight. The LAI-2000 has been used in wide range of plant canopies: coniferous and deciduous species (Gower and Norman, 1991), different pines (Smolander and Stenberg, 1996; Law et al., 2001), different species in Africa (Privette et al., 2002), Norway spruces in Germany (Küßner and Mosandl, 2000), boreal forest in Canada (Chen et al., 1997), crops (Rudorff et al., 1996 ) and weed species (Thevathasan et al., 2000). Many of these studies showed that the LAI-2000 generally underestimates the LAI from direct measurements, especially in coniferous stands. As the LAI-2000 measures the effective LAI through making the assumption of a random spatial distribution of leaves, the TRAC
(Tracing Radiation and Architecture of Canopies; 3rd Wave Engineering, Ontario, Canada) provides complementary measurements to convert the effective LAI to the true LAI (Chen, 1996). The TRAC instrument quantifies the effect of canopy architecture on optical measurements of LAI through measuring canopy gap size distribution under direct light conditions. As these two instruments are complementary, the combined use of them is suggested (Chen et al., 1997). It becomes logistically difficult to use both of them since one site has to be visited twice to get the proper lighting condition for each instrument. It is often highly desirable in a field program to collect a large sample of plots within a given period of time by using LAI-2000 at the same time as TRAC under direct sunlight conditions. The following questions therefore arise: (1) how much error will occur by using LAI-2000 under sunny conditions and (2) is it possible to make a correction for the error? The main purpose of this paper is to answer these questions. However, the importance of the work goes beyond this practical logistic constraint because the error in optical LAI measurements due to light scattering within plant canopies is widely recognised (e.g. Stenberg et al., 1994) but so far has not been systematically investigated. For this purpose, a series of measurements of LAI-2000 were taken at different sites and different solar zenith angles. A correction scheme is developed based on the solar zenith angle $\left(\theta_{\mathrm{s}}\right)$. A canopy radiative transfer model named "Five-scale" (Chen and Leblanc, 1997; Leblanc and Chen, 2000) is used to investigate the blue light scattering processes causing the errors in LAI-2000 measurements and to improve the universality of the correction scheme. Although the correction scheme is specifically derived for the LAI-2000 instrument, it can also be adopted for other instruments such as fish-eye photographing devices.

\section{Methodology}

\subsection{Gap analysis theory}

The technique of foliage estimation with optical instruments relies on measurements of the canopy gap fraction. For the LAI-2000, the gap fraction is measured by comparing the incoming diffuse radiation to the radiation measured under the canopy at five 
concentric rings: $0-13^{\circ}, 16-28^{\circ}, 32-43^{\circ}, 47-58^{\circ}$ and $61-74^{\circ}$ (LI-COR, 1991). The LAI derived from the gap fraction is defined here as the effective LAI because it is related to the solar radiation interception without the distinction between woody and photosynthetically active materials and is calculated assuming a random spatial distribution of the foliage by inverting Beer's law:

$P(\theta)=\mathrm{e}^{-G(\theta) L_{\mathrm{e}} / \cos \theta}$,

where $P(\theta)$ is the gap fraction, $G(\theta)$ the projection coefficient, $L_{\mathrm{e}}$ the effective LAI and $\theta$ the view zenith angle. However, plant canopies, such as forests, often have non-random foliage distributions. To consider foliage clumping effects, Eq. (1) can be re-written as (Nilson, 1971):

$P(\theta)=\mathrm{e}^{-G(\theta) \Omega L_{\mathrm{t}} / \cos \theta}$,

where $\Omega$ is the clumping index of the foliage at all scales and $L_{\mathrm{t}}$ the plant area index including LAI and non-green materials in the canopy. LAI is then calculated as

$L=L_{\mathrm{t}}(1-\alpha)=\frac{L_{\mathrm{e}}(1-\alpha)}{\Omega}$,

where $L$ is the LAI, $\alpha$ the woody-to-total area ratio representing the woody material contribution to $L_{\mathrm{t}}$. For conifer stands, the clumping index is written as $\Omega=\Omega_{\mathrm{E}} / \gamma_{\mathrm{E}}$, to take into account the clumping inside shoots: $\gamma_{\mathrm{E}}$ considers the clumping of needles within shoots and $\Omega_{\mathrm{E}}$ considers the clumping at scale larger than the shoots, or larger than leaves for deciduous species. $\Omega_{\mathrm{E}}$ can be measured with TRAC (Chen and Cihlar, 1995). The clumping index can vary with view zenith angle, but Kucharik et al. (1997) suggested using a clumping from $35^{\circ}$, which is the average angle of the LAI-2000 rings. However, measurements made with TRAC do not always show the large variations modelled by Kucharik et al. (1998). Moreover, simulations done with Five-scale have also shown that the variation is often very small over the $20-70^{\circ}$ range and that the mean clumping index between 30 and $60^{\circ}$ is very close to the mean clumping index of all angles. Because of the foliage orientation, one single view zenith angle measurement cannot be used without information or assumption about $G(\theta)$, therefore the effective LAI is found with (Miller, 1967):
$L_{\mathrm{e}}=2 \int_{0}^{\pi / 2} \ln \left[\frac{1}{P(\theta)}\right] \cos \theta \sin \theta \mathrm{d} \theta$.

The computation of $L_{\mathrm{e}}$ with Eq. (4) using the LAI-2000 is a discrete summation over the five view zenith angles using $\theta$ from the centre of each ring. Under the assumption of random leaf angle distribution (i.e. $G(\theta)=0.5$ ), $L_{\mathrm{e}}$ can be calculated for each view zenith angle. Since the multiple scattering of blue light is not expected to be the same at all angles, we will examine the options of retrieving $L_{\mathrm{e}}$ with Eq. (4) and from each ring separately. The five rings of the LAI-2000 do not cover the full $90^{\circ}$ range, and the C2000 software (LI-COR, 1991) given with the instruments assumes that the fifth ring that covers the range of view zenith angle from 61 to $74^{\circ}$ has a $\mathrm{d} \theta$ of $29^{\circ}$ which covers the range $61-90^{\circ}$ (LI-COR, 1991). This further increases the weight for the fifth ring that is already larger than any other rings because of the $\sin \left(68^{\circ}\right)$. We use $\mathrm{d} \theta$ as the angular range of each rings in this paper. This gives a systematic increase of $8 \%$ in $L_{\mathrm{e}}$ for all cases as compared with the $\mathrm{C} 2000$ software calculation.

The TRAC can also measure the gap fraction, but only at the solar zenith angle. TRAC is an optical instrument that measures the photosynthetic photon flux density (PPFD) through a canopy at a high frequency of $32 \mathrm{~Hz}$ (Chen and Cihlar, 1995). By walking the instrument under a canopy, its gap fraction and gap size distribution can be computed once the penumbra effect has been considered. The gap fraction is used to estimate the effective LAI, while the gap size distribution is used to estimate the clumping index. The clumping index is found by comparing the measured gap size distribution with a theoretical gap size distribution associated with a canopy with randomly distributed foliage elements (Chen and Cihlar, 1995):

$\Omega_{\mathrm{E}}=\frac{\left[1+\left(F_{\mathrm{m}}-F_{\mathrm{mr}}\right)\right] \ln \left[F_{\mathrm{m}}\right]}{\ln \left[F_{\mathrm{mr}}\right]}$,

where $F_{\mathrm{m}}$ is the measured total canopy gap fraction and $F_{\mathrm{mr}}$ the gap fraction for the same canopy, where the foliage elements are computationally re-arranged to become spatially random. $F_{\mathrm{m}}$ can be measured as the transmittance of direct or diffuse radiation at the zenith angle of interest, while $F_{\mathrm{mr}}$ is found from the measured gap size distribution through a gap removal approach. 


\subsection{Site description and experimental procedure}

Larose forest is situated about $45 \mathrm{~km}$ east of Ottawa, Ont. and has an area of about $108 \mathrm{~km}^{2}$. It is privately owned but administrated by the Ontario provincial government that is in charge of timber harvest and re-plantation. An old inventory (Reid, 1979) of the forest mentioned $47.9 \%$ coverage by deciduous species, mainly aspens (Populus tremuloides), with some paper birches (Betula papyrifera) and sugar maple trees (mainly Acer saccharum). The rest is occupied by conifer species including white spruces (Picea glauca) $(27.6 \%)$, red pines (Pinus resinosa) (13.5\%), and white pines (Pinus strobus) (9.3\%), and others $(1.8 \%)$. Most of the deciduous trees were severely damaged during a 5-day freezing-rain storm in January 1998, but the coniferous survived with little damage: only a few branches and trunks broke and fell on the ground. The measurements used in this study were taken the year before the ice storm for the deciduous sites and after the ice storm in conifer sites that suffered minimal damage.

From May 1 to November 20, 1997, the Larose forest was visited several times in order to measure the seasonal gap fraction and LAI variations of two deciduous sites. Site D1 $\left(45^{\circ} 23^{\prime} 21^{\prime \prime} \mathrm{N}, 75^{\circ} 11^{\prime} 19^{\prime \prime} \mathrm{W}\right)$ is about $70 \%$ poplar (mainly P. tremuloides with a few Populus balsamifera L.) with DBH from 15 to $45 \mathrm{~cm}$, $20 \%$ birch and $10 \%$ of other trees including sugar and red maples (Acer rubrum L.), and American elms (Ulmus Americana L.). Site D1 has a $90 \mathrm{~m}$ transect in the east-west direction and no definite boundary in the north-south direction, but it is bounded by the instrument footprint estimated at about $\pm 30 \mathrm{~m}$ from the transect. Site D2 $\left(45^{\circ} 22^{\prime} 49^{\prime \prime} \mathrm{N}, 75^{\circ} 11^{\prime} 48^{\prime \prime} \mathrm{W}\right)$ has a $60 \mathrm{~m}$ transect and is mainly composed of aspen trees (80\%) with maximum DBH around $25 \mathrm{~cm}$ with most trees DBH between 10 and $15 \mathrm{~cm}$. The remaining 20\% are composed of birch, and maple trees. Site D2 is a younger and more homogeneous site than D1.

The conifer sites are plantations of singular species. Site P1 $\left(45^{\circ} 2^{\prime} 40^{\prime \prime} \mathrm{N}, 75^{\circ} 09^{\prime} 05^{\prime \prime} \mathrm{W}\right)$ is composed of red pine with DBH from 25 to $30 \mathrm{~cm}$, while site P2 $\left(45^{\circ} 23^{\prime} 55^{\prime \prime} \mathrm{N}, 75^{\circ} 07^{\prime} 13^{\prime \prime} \mathrm{W}\right)$ is composed of white pines with DBH between 20 and $30 \mathrm{~cm}$. No permanent transects were deployed in the pine sites. Because of its small extent, two short transects of $30 \mathrm{~m}$ were deployed in $\mathrm{P} 1$, while a transect of $100 \mathrm{~m}$ was used for $\mathrm{P} 2$.
Flags were displayed every $10 \mathrm{~m}$ along the transects. LAI-2000 measurements were taken using a $270^{\circ}$ view restrictor at each of these flags (e.g. 10 flags for site D1 and seven flags for D2). Having flags systematically every $10 \mathrm{~m}$ allows a non-bias sampling of the canopy. The choice of transects at each site was made compatible with the TRAC instrument requirements: TRAC transects are preferred to be perpendicular to the sun's azimuth. Two LAI-2000 reference measurements for the above canopy value were taken in an open area near the sites, one before and another one after each site with the same instruments used above the canopy. The below-canopy and reference measurements were all taken facing from north-east to north-west where the opening had the largest extent. The two reference measurements were about $30 \mathrm{~min}$ apart for all measurements in this paper. The reference used for each below-canopy comparison is a linear interpolation (in time) between the two reference values. The interpolation was important when the measurements were taken near sunset or sunrise since the amount of radiation reaching the canopy changed rapidly.

The TRAC was walked along the transects starting from the most eastern flag, measuring gaps with a resolution of about $1 \mathrm{~cm}$. Each time one of the flag was reached, a marker was entered in the data stream to compute the actual measurement resolution between two flags. All segments for each site were analysed together using the TRACWin software provided with the instrument (Leblanc et al., 2001). PPFD references were acquired in a large opening just before or after each transect to assure that the maximum PPFD is known. TRAC measurements were acquired during the afternoon of September 5, 1997 with the solar zenith angle at 40.5 and $52.2^{\circ}$ for D1, and 41.2 and $54.9^{\circ}$ for D2. TRAC measurements at P1 were done on July 31, 1998 , so a smaller solar zenith angle of about $27^{\circ}$ was possible, while the measurements for P2 were done on August 12, 1999 with solar zenith angles ranging from 37.2 to $71.0^{\circ}$.

\section{Results and discussion}

\subsection{Seasonal variation of effective LAI}

Fig. 1 shows the $L_{\mathrm{e}}$ seasonal variation based on LAI-2000 gap fraction measurements using Eq. (4) 


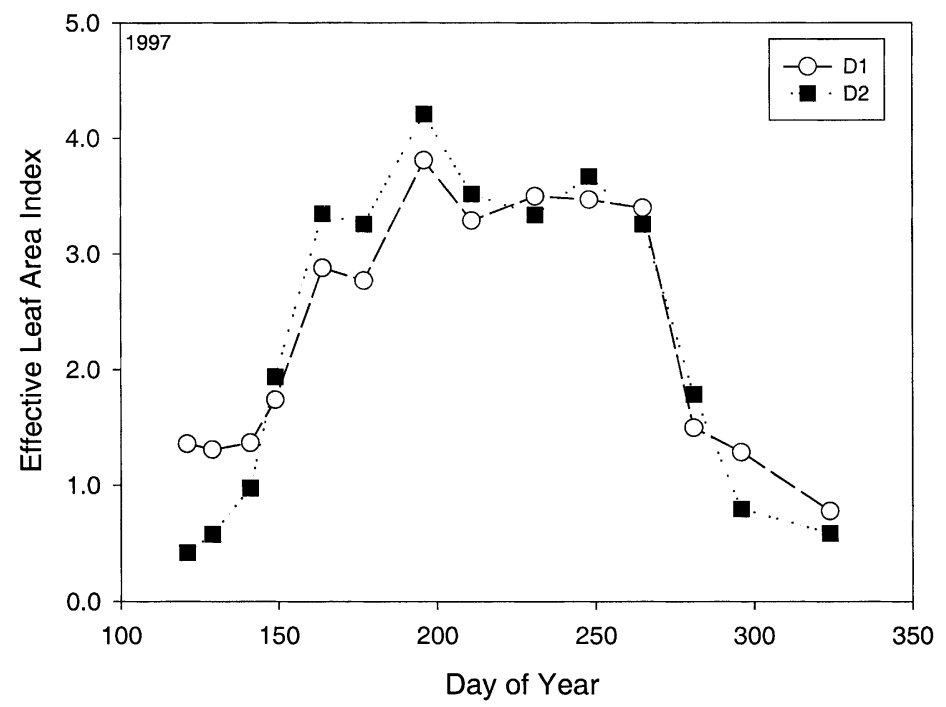

Fig. 1. Seasonal variation of effective LAI for two deciduous sites in Larose forest based on gap fraction measured with the LAI-2000 in 1997.

for the deciduous sites. The numerical values can be seen in Table 1. Following the LAI-2000 operating manual, the measurements were taken under diffuse light, except for 3 days where the measurements were taken under sunny conditions: June 26 (day of year 177), July 30 (day of year 211) and August 19 (day of year 231). For these 3 days, the measurements were done with the sensor placed in the opposite direction to the sun (i.e. with the sun behind the operator and sensor in front of the operator), and the $270^{\circ}$ view restrictor was used to block off the shadow of the operator and any possible influence of the direct light on the sensor. The "above-canopy" reference measurements made outside the canopy were made in the same way. The last measurements, taken on November 20, were made with snow on the ground, but the trees were free of snow. The important difference between the $L_{\mathrm{e}}$ measured in May and November in site D1, when no foliage was present in either case, is due mainly to a few trees that were cut by beavers during the summer in the field of view of the LAI-2000. From Fig. 1, it is clear that the measurements taken under sunlit conditions are well below the other values measured during the peak of the growing season.

\subsection{Effective LAI variation with solar zenith angle}

Effective LAI measurements taken on September 22, 1997 from 2 to 7 p.m. (local summer time: GMT-4) in both the deciduous sites are used to study the influence of direct radiation on LAI-2000 measurements. This day was cloudless during the measurements; some clouds appeared near sunset. The last measurements, taken just before 7 p.m. near sunset, were taken under a complete diffuse light condition. The same experiment was undertaken on August 27, 1998, in P1 and on August 12, 1999, in $\mathrm{P} 2$. Table 2 has the measurements from the deciduous and coniferous sites at different solar zenith angles.

In order to make our study statistically significant, the four sites are used in the analysis to increase the sample size. Because each site has a different $L_{\mathrm{e}}$, the relative change in $L_{\mathrm{e}}$ is studied by taking the ratio of the effective LAI calculated with Eq. (4) at a specific $\theta_{\mathrm{s}}$, denoted $L_{\mathrm{e}}\left(\theta_{\mathrm{s}}\right)$, and the "real" $L_{\mathrm{e}}$. The real $L_{\mathrm{e}}$ is taken as the $L_{\mathrm{e}}$ values measured near sunset, which is normally the largest. The ratio $L_{\mathrm{e}}\left(\theta_{\mathrm{S}}\right) / L_{\mathrm{e}}$ is plotted against $\theta_{\mathrm{s}}$ in Fig. 2a for all sites. Fig. 2a shows that the scatter of the data points is small. This indicates that the variation in LAI estimation with the 
Table 1

Seasonal effective LAI, with illumination conditions and sun corrected $L_{\mathrm{e}}$, clumping corrected $L_{\mathrm{e}} / \Omega$ and final LAI measurements in two deciduous sites (D1 and D2) from May 1 to November 20, 1997

\begin{tabular}{|c|c|c|c|c|c|c|c|}
\hline Site & Day number & Date & $L_{\mathrm{e}}(\mathrm{LAI}-2000)$ & Illumination condition & Sun corrected, $L_{\mathrm{e}}$ & $L_{\mathrm{e}} / \Omega$ & LAI \\
\hline \multirow[t]{14}{*}{ D1 } & 121 & 1 May 1997 & 1.36 & Diffuse light & 1.36 & 1.46 & 0.00 \\
\hline & 129 & 9 May 1997 & 1.31 & Diffuse light & 1.31 & 1.41 & 0.01 \\
\hline & 141 & 21 May 1997 & 1.37 & Diffuse light & 1.37 & 1.47 & 0.11 \\
\hline & 149 & 29 May 1997 & 1.74 & Diffuse light & 1.74 & 1.87 & 0.53 \\
\hline & 164 & 13 June 1997 & 2.88 & Diffuse light & 2.88 & 3.10 & 1.80 \\
\hline & 177 & 26 June 1997 & 2.77 & $\theta_{\mathrm{s}}: 31.1^{\circ}$ & 3.18 & 3.41 & 2.16 \\
\hline & 196 & 15 July 1997 & 3.81 & Diffused light & 3.81 & 4.10 & 2.89 \\
\hline & 211 & 30 July 1997 & 3.29 & $\theta_{\mathrm{S}}: 52.4^{\circ}$ & 3.68 & 3.96 & 2.80 \\
\hline & 231 & 19 August 1997 & 3.50 & $\theta_{\mathrm{s}}: 67.3^{\circ}$ & 3.82 & 4.11 & 3.00 \\
\hline & 248 & 5 September 1997 & 3.47 & Diffuse light & 3.47 & 3.73 & 2.68 \\
\hline & 265 & 22 September 1997 & 3.40 & Diffuse light & 3.40 & 3.66 & 2.65 \\
\hline & 281 & 8 October 1997 & 1.50 & Diffuse light & 1.50 & 1.61 & 0.65 \\
\hline & 296 & 23 October 1997 & 1.29 & Diffuse light & 1.29 & 1.39 & 0.48 \\
\hline & 324 & 20 November 1997 & 0.78 & Diffuse light & 0.78 & 0.84 & 0.00 \\
\hline \multirow[t]{14}{*}{ D2 } & 121 & 1 May 1997 & 0.42 & Diffuse light & 0.42 & 0.46 & 0.00 \\
\hline & 129 & 9 May 1997 & 0.58 & Diffuse light & 0.58 & 0.63 & 0.17 \\
\hline & 141 & 21 May 1997 & 0.98 & Diffuse light & 0.98 & 1.07 & 0.60 \\
\hline & 149 & 29 May 1997 & 1.94 & Diffuse light & 1.94 & 2.11 & 1.63 \\
\hline & 164 & 13 June 1997 & 3.35 & Diffuse light & 3.35 & 3.64 & 3.15 \\
\hline & 177 & 26 June 1997 & 3.26 & $\theta_{\mathrm{s}}: 40.1^{\circ}$ & 3.71 & 4.03 & 3.52 \\
\hline & 196 & 15 July 1997 & 4.21 & Diffuse light & 4.21 & 4.57 & 4.05 \\
\hline & 211 & 30 July 1997 & 3.52 & $\theta_{\mathrm{s}}: 57.6^{\circ}$ & 3.91 & 4.25 & 3.71 \\
\hline & 231 & 19 August 1997 & 3.34 & $\theta_{\mathrm{s}}: 62.9^{\circ}$ & 3.67 & 3.99 & 3.44 \\
\hline & 248 & 5 September 1997 & 3.67 & Diffuse light & 3.67 & 3.99 & 3.42 \\
\hline & 265 & 22 September 1997 & 3.26 & Diffuse light & 3.26 & 3.54 & 2.96 \\
\hline & 281 & 8 October 1997 & 1.79 & Diffuse light & 1.79 & 1.95 & 1.35 \\
\hline & 296 & 23 October 1997 & 0.80 & Diffuse light & 0.80 & 0.87 & 0.26 \\
\hline & 324 & 20 November 1997 & 0.59 & Diffuse light & 0.59 & 0.64 & 0.00 \\
\hline
\end{tabular}

LAI-2000 under the presence of direct sunlight may not be species dependent. The following exponential function was used to fit the data

$\frac{L_{\mathrm{e}}\left(\theta_{\mathrm{s}}\right)}{L_{\mathrm{e}}}=A+B \mathrm{e}^{C \theta_{\mathrm{s}}}$,

where $A, B$, and $C$ are coefficients found through the empirical fit and $\theta_{\mathrm{s}}$ the solar zenith angle in degrees. The coefficient of determination $R^{2}$ of the regression was found to be 0.41 . Since the measurements were taken with the sensor head oriented away from the sun with a $270^{\circ}$ view restrictor and that the sun position changes during the course of the day, each point in Fig. 2a was taken with a slightly different view of the canopy. This may have introduced some $L_{\mathrm{e}}$ variations since the sites were not completely azimuthally homogeneous. In some cases, the above-canopy reference measurements made in an opening can also introduce errors when the openness of the reference field is limited in some directions. The white pine site (P2) may have suffered from this type of errors. Its reference area, which is a road crossing just outside the stand, was adequate in the afternoon, but once the sun moved further west, the reference was influenced by the edge of the forest. Because of this, effective LAI measurements in P2 near sunset are smaller than previous measurements taken during that day.

The correction scheme determined from the four sites was applied to the measurements of both deciduous sites of the Larose forest for 3 days when the measurements were taken under sunny conditions. Once corrected, the variations in the measured $L_{\mathrm{e}}$ values for the two sites during the summer are much reduced (see Table 1 and Fig. 3). This is expected since the amount of foliage should have not changed much during July and August. 

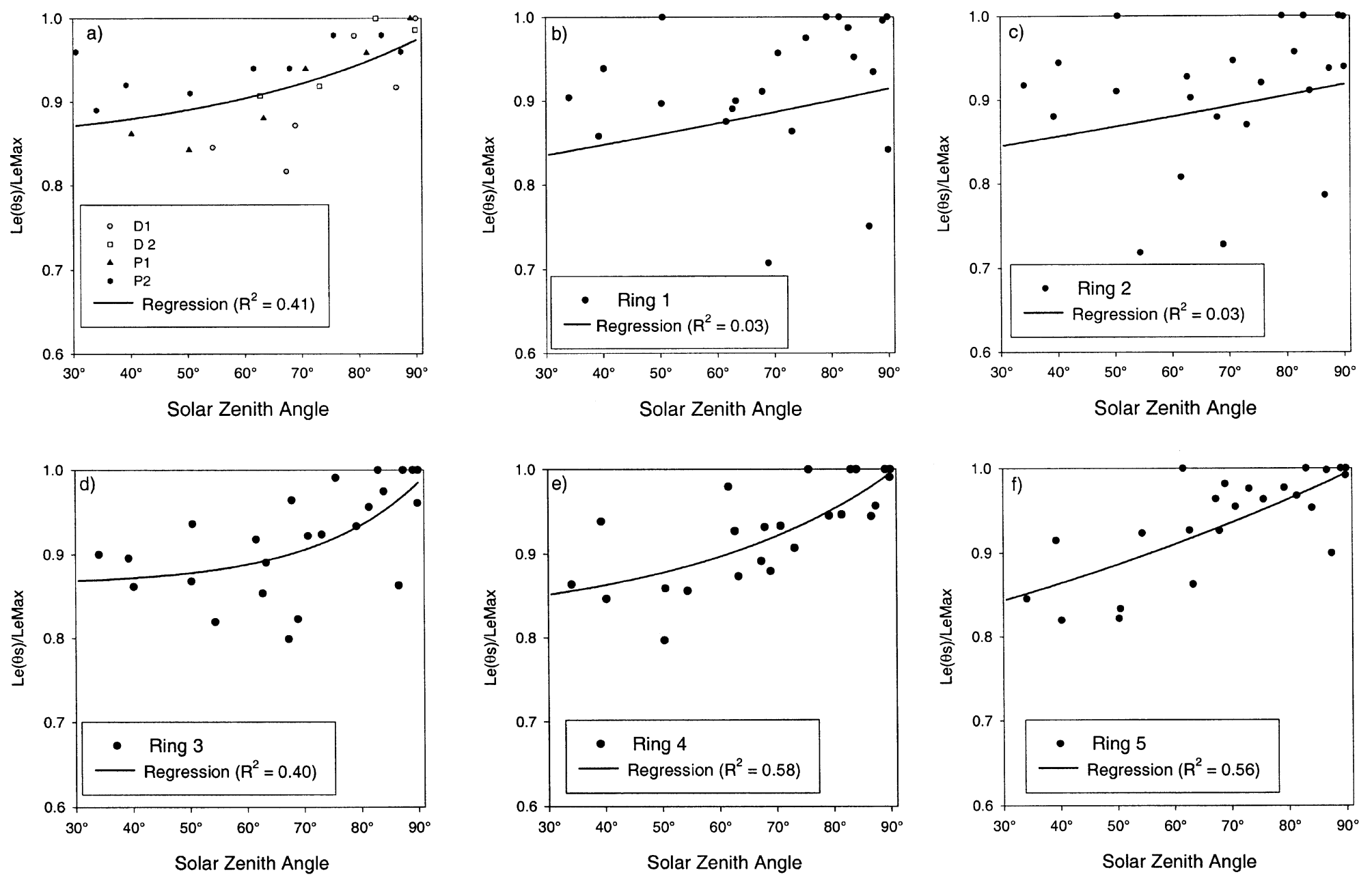

Fig. 2. Ratio of effective LAI taken at different solar zenith angles $\left(L_{\mathrm{e}}\left(\theta_{\mathrm{s}}\right)\right)$ to the maximum effective LAI found at large solar zenith angles $\left(L_{\mathrm{e}}(\mathrm{max})\right)$ for $(\mathrm{a})$ all LAI-2000 rings, (b) ring 1 , (c) ring 2 , (d) ring 3 , (e) ring 4 and (f) ring 5 . 
Table 2

Effective LAI measurements under sunlit conditions for four sites in Larose forest

\begin{tabular}{|c|c|c|c|c|c|}
\hline Site & Day number & Date & $L_{\mathrm{e}}(\mathrm{LAI}-2000)$ & $\theta_{\mathrm{s}}\left(^{\circ}\right)$ & $L_{\mathrm{e}}\left(\theta_{\mathrm{s}}\right) / L_{\mathrm{e}}$ \\
\hline \multirow[t]{6}{*}{ D1 } & 265 & 22 September 1997 & 2.87 & 54.4 & 0.85 \\
\hline & & & 2.77 & 67.3 & 0.82 \\
\hline & & & 2.96 & 68.9 & 0.87 \\
\hline & & & 3.33 & 79.2 & 0.98 \\
\hline & & & 3.12 & 86.6 & 0.92 \\
\hline & & & 3.40 & 90.0 & 1.00 \\
\hline \multirow[t]{4}{*}{ D2 } & 265 & 22 September 1997 & 3.00 & 62.7 & 0.91 \\
\hline & & & 3.04 & 73.1 & 0.92 \\
\hline & & & 3.30 & 83.0 & 1.00 \\
\hline & & & 3.26 & 89.9 & 0.99 \\
\hline \multirow[t]{6}{*}{ P1 } & 239 & 27 August 1998 & 2.43 & 40.2 & 0.86 \\
\hline & & & 2.37 & 50.3 & 0.84 \\
\hline & & & 2.48 & 63.3 & 0.88 \\
\hline & & & 2.64 & 70.7 & 0.94 \\
\hline & & & 2.70 & 81.4 & 0.96 \\
\hline & & & 2.82 & 89.1 & 1.00 \\
\hline \multirow[t]{8}{*}{$\mathrm{P} 2$} & 224 & 12 August 1999 & 2.89 & 34.1 & 0.89 \\
\hline & & & 2.99 & 39.3 & 0.92 \\
\hline & & & 2.97 & 50.5 & 0.91 \\
\hline & & & 3.07 & 61.6 & 0.94 \\
\hline & & & 3.05 & 67.9 & 0.94 \\
\hline & & & 3.20 & 75.6 & 0.98 \\
\hline & & & 3.17 & 84.0 & 0.98 \\
\hline & & & 3.12 & 87.4 & 0.96 \\
\hline
\end{tabular}

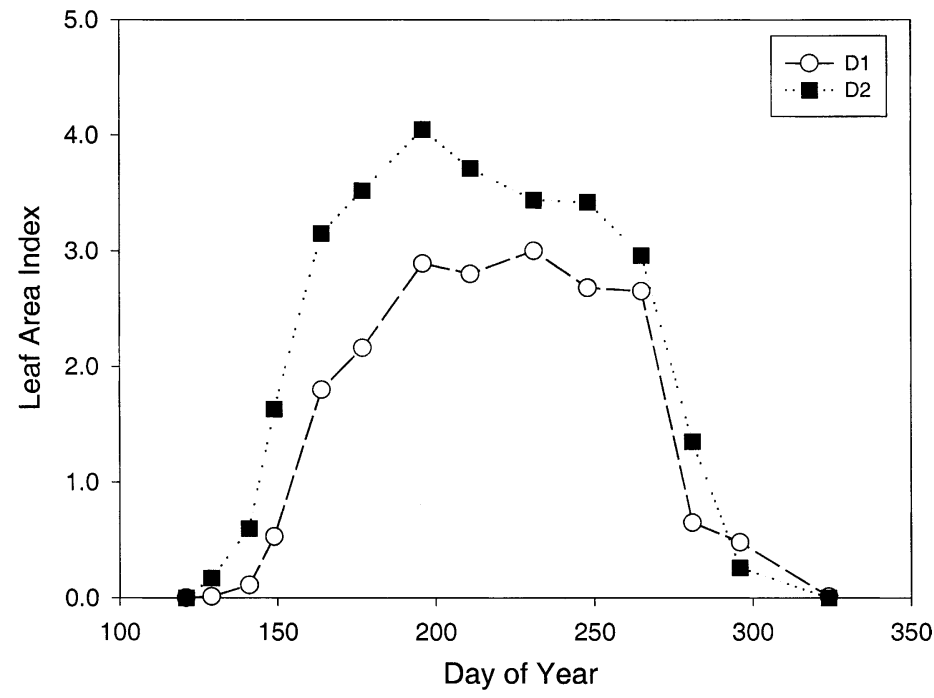

Fig. 3. Seasonal variation of LAI for two deciduous sites based on the effective LAI from Fig. 1, corrected for blue light scattering, foliage clumping and woody material. 
Table 3

Clumping index and effective LAI measurements with TRAC, and effective LAI from LAI-2000 all rings and with the fourth ring only

\begin{tabular}{lllllll}
\hline TRAC & TRAC $L_{\mathrm{e}}\left(\theta_{\mathrm{s}}\right)$ & TRAC $\Omega_{\mathrm{E}}\left(\theta_{\mathrm{s}}\right)$ & $\theta_{\mathrm{s}}\left({ }^{\circ}\right)$ & TRAC $\left(L_{\mathrm{e}}\right)$ & LAI-2000 $\left(L_{\mathrm{e}, \text { all }}\right)$ & LAI-2000 $\left(L_{\mathrm{e}, 4}\right)$ \\
\hline D1 (5 September 1997) & 4.54 & 0.96 & 40.5 & 4.05 & 3.40 & 3.63 \\
& 3.64 & 0.91 & 52.2 & & & 3.42 \\
D2 (5 September 1997) & 3.66 & 0.86 & 41.2 & 3.61 & 3.20 & 2.96 \\
& 3.56 & 0.97 & 54.9 & & \\
P1 (31 July 1998) & 2.72 & 0.68 & 26.8 & 2.80 & & 3.30 \\
& 2.87 & 0.77 & 26.9 & & 3.20 \\
P2 (12 August 1999) & 4.19 & 0.84 & 37.2 & 3.25 & & \\
& 2.84 & 0.90 & 62.9 & & & \\
\hline
\end{tabular}

Using the $L_{\mathrm{e}}$ measurements at the beginning of the season, on May 1, when no foliage was present, the amount of woody material can be estimated. It was used to remove the effect of woody materials on LAI measurements. Eq. (3) can then be re-written as

$L=\frac{L_{\mathrm{e}}}{\Omega}-a$

where $a$ is the woody area index measured before leaf-on or after leaf-off.

The clumping index $\Omega$ was computed from TRAC measurements made on September 5, 1997. The clumping index was 0.93 and 0.92 for site D1 and D2, respectively (Table 3 has the TRAC measurements used). Such values of the clumping index near unity indicate that the foliage in both sites is almost randomly distributed. Fig. 3 has the final LAI values corrected for blue light scattering, clumping, and woody material. The younger site (D2) final LAI values show that it has more green foliage than the older site (D1).

\subsection{General correction based on the LAI-2000 fourth ring}

The correction shown in Eq. (6) found through empirical fit allowed the LAI taken under sunny conditions to be more consistent with other measurements taken under diffuse conditions. The universality of this equation relies here on 24 measurements from four sites. This does not constitute a large enough sampling to claim that the correction can be used for any other sites, especially with a coefficient of determination of only 0.41 . Table 4 shows $R^{2}$ much larger when the correlation is done for each site separately, but at the expense of having fewer data points. The values of $A$, $B$, and $C$ found through the fits are very similar for site $\mathrm{D} 1, \mathrm{D} 2$ and $\mathrm{P} 1$. The range of effective LAI of our sites was not very large, from three to a little more than four. But each of the sites exhibits different leaf orientation that makes them unique and different. Since the influence of the sun may be different in each ring, measurements of the effective LAI using Eq. (4) may be contaminated differently by reflected solar radiation directly from the foliage to the LAI-2000 sensor. This leads to the separation of the influence of solar zenith angle on the measurements of $L_{\mathrm{e}}$ in the five rings (Fig. 2b-f).

Fig. $2 b-f$ are based on the same measurements as Fig. 2a, but separated by view rings. An assumption of

Table 4

Coefficients $A, B$, and $C$ and coefficient of determination $R^{2}$ for Eq. (6) for the four sites separately in Larose forest and for all sites together for all LAI-2000 rings and for each ring separately

\begin{tabular}{lllll}
\hline & $A$ & $B$ & $C$ & $R^{2}$ \\
\hline All rings & & & & \\
D1 & 0.778 & 0.0062 & 0.039 & 0.68 \\
D2 & 0.835 & 0.010 & 0.030 & 0.78 \\
P1 & 0.799 & 0.014 & 0.030 & 0.93 \\
P2 & 0.466 & 0.389 & 0.003 & 0.83 \\
All sites & & & & \\
All rings & 0.842 & 0.0139 & 0.025 & 0.41 \\
Ring 1 & 0.462 & 0.339 & 0.003 & 0.03 \\
Ring 2 & 0.522 & 0.292 & 0.003 & 0.03 \\
Ring 3 & 0.864 & 0.001 & 0.053 & 0.40 \\
Ring 4 & 0.815 & 0.017 & 0.026 & 0.58 \\
Ring 5 & 0.590 & 0.201 & 0.008 & 0.56 \\
\hline
\end{tabular}


random orientation of foliage is used to retrieve $L_{\mathrm{e}}$ using individual rings (i.e. $G(\theta)=0.5$ ). It is clear from the $R^{2}$ values that the ratio $L_{\mathrm{e}}\left(\theta_{\mathrm{s}}\right) / L_{\mathrm{e}}$ from the rings at large view zenith angles $\left(32-74^{\circ}\right)$ have a stronger relationship with $\theta_{\mathrm{s}}$ than rings one and two $\left(0-28^{\circ}\right)$. Table 4 shows the regression parameters associated with Fig. 2. Ring four has the largest $R^{2}$ with 0.58 . The relative r.m.s. error is $4 \%$.

Another way to display the effective LAI retrieval dependency on the rings and solar zenith angles is given in Fig. $4 \mathrm{a}-$ d. $L_{\mathrm{e}}$ measurements taken at different $\theta_{\mathrm{s}}$ for each individual ring are plotted by sites. The first feature to notice is the lack of variability of
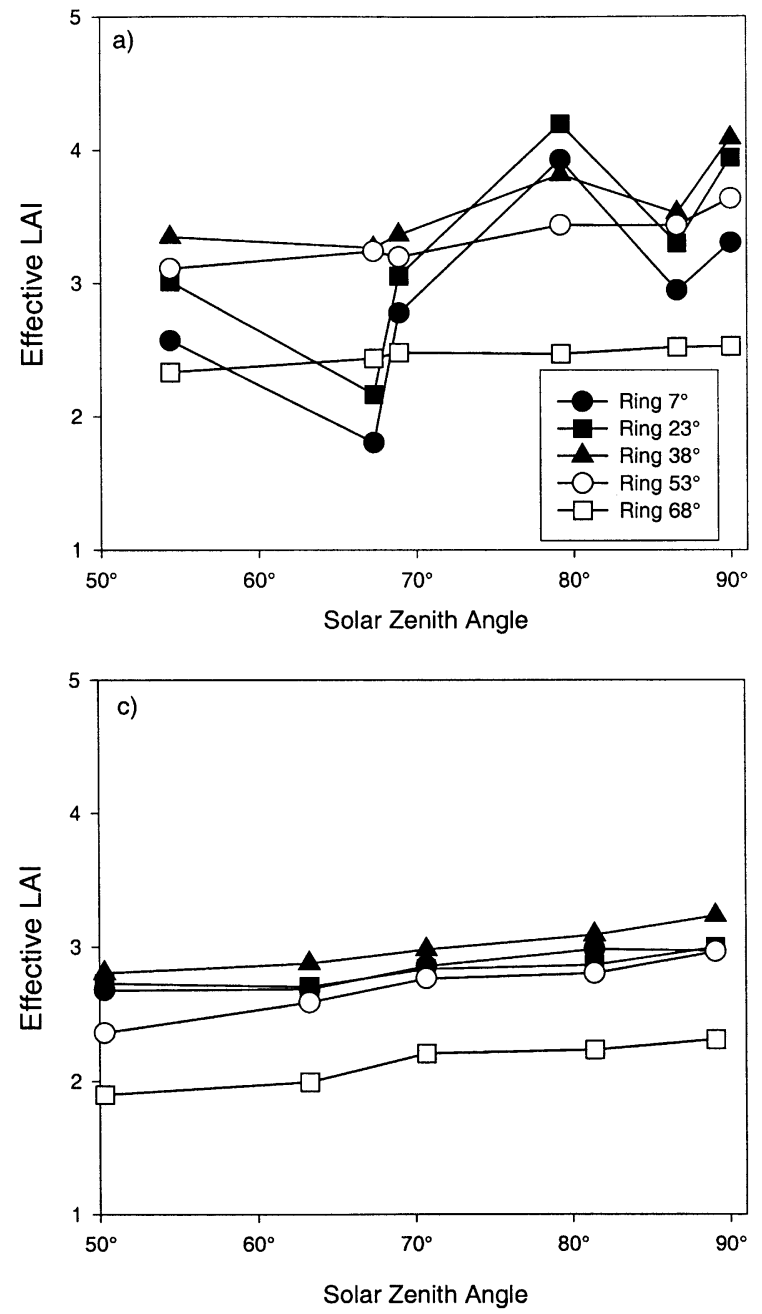

the fifth ring $\left(68^{\circ}\right)$ as the $\theta_{\mathrm{s}}$ varies from site to site. The $L_{\mathrm{e}}$ based on the fifth ring measurements always gave a value near two. This seems to indicate that the fifth ring is not very sensitive to changes in canopy $L_{\mathrm{e}}$. This could be caused by a combination of the small gap fractions and relatively large multiple scattering in that ring. Fig. 4a and $d$ also suggest that the first and second rings are very unstable, probably more susceptible to sky radiation variation because of large openings in the canopy or because of the effects of sunlit leaves viewed by the sensor, especially near zenith (ring one). The third and fourth rings seem to be the most stable rings at all sites. This could imply that
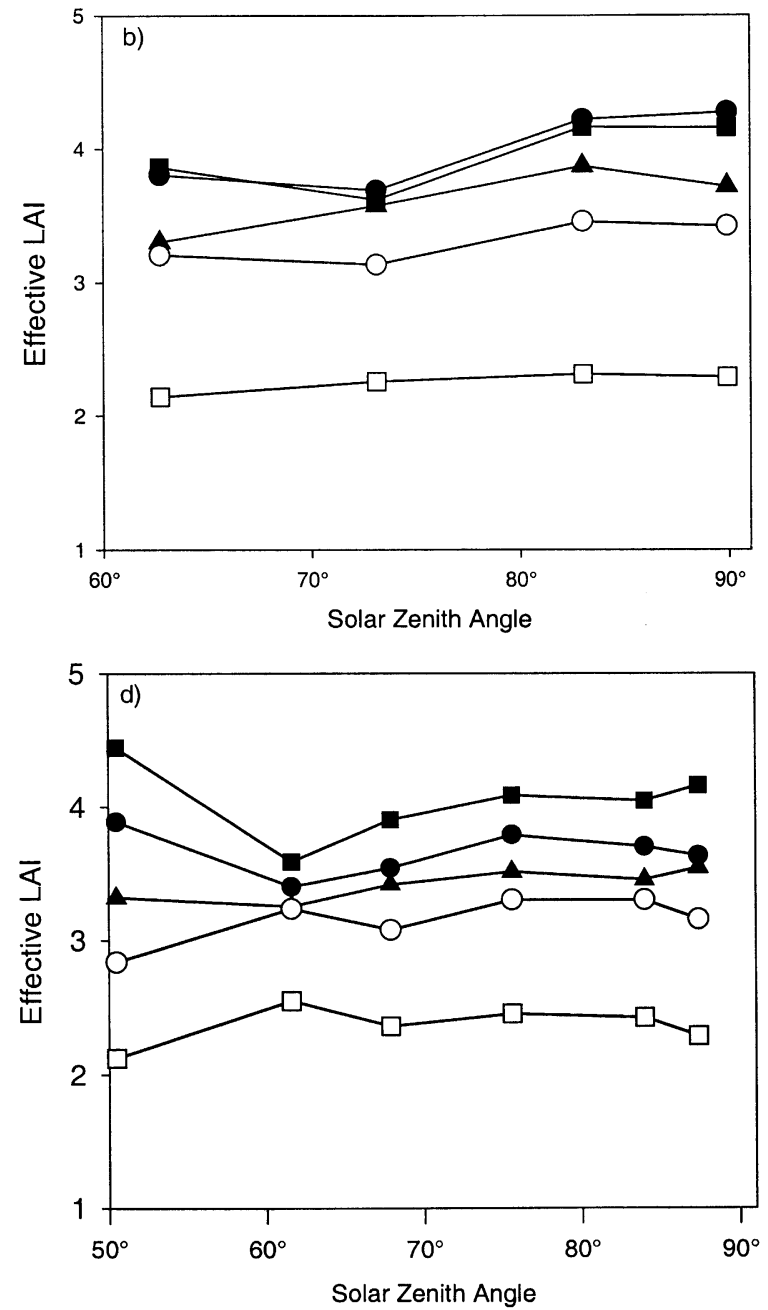

Fig. 4. Effective LAI measurements taken at different solar zenith angles separated by rings for (a) D1, (b) D2, (c) P1 and (d) P2 sites. 
in the sun's presence, only the third and fourth rings should be used to calculate the effective LAI. Based on model simulations, Chen and Black (1991) found that at a zenith angle of $62^{\circ}$ all leaf and branch inclinations give a convergence point where $G(\theta)=0.54$ for clumped canopies. Warren-Wilson (1965) showed that $G\left(57.5^{\circ}\right)=0.5$ for random canopies. This implies that a random foliage assumption near $60^{\circ}$ is reasonable and that using $G(\theta)=0.5$ at $53^{\circ}$ should not induce large errors in the effective LAI estimation.

To assess the dependence of the effective LAI measurements on solar zenith angle, the radiative transfer model Five-scale (Chen and Leblanc, 1997; Leblanc and Chen, 2000) is used to simulate the diffuse radiation measured by the LAI-2000 under a canopy. Five-scale was developed with emphasis on the structural composition of forest canopies at different scales: crowns, group of crowns, branches and shoots. Five-scale's multiple scattering scheme (Chen and Leblanc, 2001), which considers canopy geometrical effects on all orders of multiple scattering, is used here to simulate the amount of blue radiation reaching the LAI-2000 sensor under various sky conditions.

The diffuse radiation measured by the LAI- 2000 can be separated into two components: diffuse sky radiation that reaches the sensor through gaps in the canopy, and enhanced diffuse radiation due to multiple scattering of both the direct and diffuse radiation within the canopy. When the measurements are taken under diffuse sky conditions, the multiple scattering inside the canopy is small, but when the sun directly illuminates the canopy, the sensor receives more radiation than that from the sky through canopy gaps (Welles and Norman, 1991). The largest source of that radiation is from sunlit foliage, but the rest of the foliage is not completely dark due to multiple scattering. The apparent gap fraction measured by the LAI-2000 can be written as

$P^{\prime}\left(\theta, \theta_{\mathrm{s}}\right)=P(\theta)+[1-P(\theta)] \frac{F_{\text {scatt }}\left(\theta, \theta_{\mathrm{s}}\right)}{F_{\text {skydiff }}\left(\theta_{\mathrm{s}}\right)}$,

where $P(\theta)$ is the real gap fraction at the view zenith angle $\theta$ in which only the diffused sky radiation passes through, $F_{\text {skydiff }}\left(\theta_{\mathrm{s}}\right)$ the sky diffuse irradiance and $F_{\text {scatt }}\left(\theta, \theta_{\mathrm{s}}\right)$ represents the irradiance reaching the LAI-2000 due to multiple scattering in the canopy. If there is not multiple scattering in the canopy, $F_{\text {scatt }}(\theta$, $\left.\theta_{\mathrm{s}}\right)$ is zero. Thus, the second term of Eq. (8) quantifies the increase from the real gap fraction due to multiple scattering in the canopy. In this study, the sky diffuse irradiance is assumed to be from Rayleigh scattering only. The canopy simulations were done with the assumption of a random foliage angle distribution, at a wavelength of $450 \mathrm{~nm}$ (blue) with effective foliage reflectivity of 0.02 . The reflectivity value includes green foliage and woody material such as trunks and branches. The transmittance at the blue wavelength is assumed to be negligible. The gap fractions computed from Eq. (8) for the five view angles of the LAI-2000 are then converted into effective LAI by inverting Eq. (1). The simulated $L_{\mathrm{e}}\left(\theta_{\mathrm{s}}\right) / L_{\mathrm{e}}$ versus $\theta_{\mathrm{s}}$ curves are shown in Fig. 5. Three simulations were done at $L_{\mathrm{e}}$ of $1,3.5$ and 7 with no clumping and negligible woody material, i.e. $\mathrm{LAI}=L_{\mathrm{e}}$. The second simulation, with $L_{\mathrm{e}}=3.5$, corresponds to the study sites of this paper. For that case, the first four rings gave a ratio $L_{\mathrm{e}}\left(\theta_{\mathrm{s}}\right) / L_{\mathrm{e}}$ in the 0.84-0.89 range at $\theta_{\mathrm{s}}=20^{\circ}$. The fifth ring has a more pronounced change in $L_{\mathrm{e}}$ with $\theta_{\mathrm{s}}$. The ratio for the fifth ring is less than 0.7 at $\theta_{\mathrm{s}}=20^{\circ}$. One interesting aspect of the other two simulations is the great variability of almost all rings with LAI. Ring four is least affected by the changes in LAI, re-enforcing the argument that this ring may be the best ring to use in the correction scheme. The large change in ring 5 $\left(61-74^{\circ}\right)$ between the cases with LAI of one (Fig. 5a) and seven (Fig. 5c) is dependant on the small gap fraction found: $P(\theta) \ll 1$ which gives a large weight to the scattering component $F_{\text {scatt }}\left(\theta, \theta_{\mathrm{s}}\right)$ in Eq. (8). On the other hand, ring one $\left(0-13^{\circ}\right)$ is very much influenced by directly viewed sunlit foliage when the foliage is sparse, which is shown clearly in Fig. 5a. As the gap fraction diminishes with increasing LAI, the sensor sees reduced sunlit foliage, and thus this influence on ring one is reduced.

Since ring four has been shown from measurements and simulations to be the most consistent in capturing multiple scattering effects at various solar zenith angles and LAI values, it is practical to chose it as a basis for a general correction scheme. Fig. 6a demonstrates that the influence of LAI on the fourth ring is still considerable, but follows some predictable patterns. It is interesting to note that all lines cross near $62^{\circ}$, the same zenith angle found by Chen and Black (1991), where the extinction coefficients of foliage and branches at various inclination angles differ the least. Using this angle knowledge, the following expression 

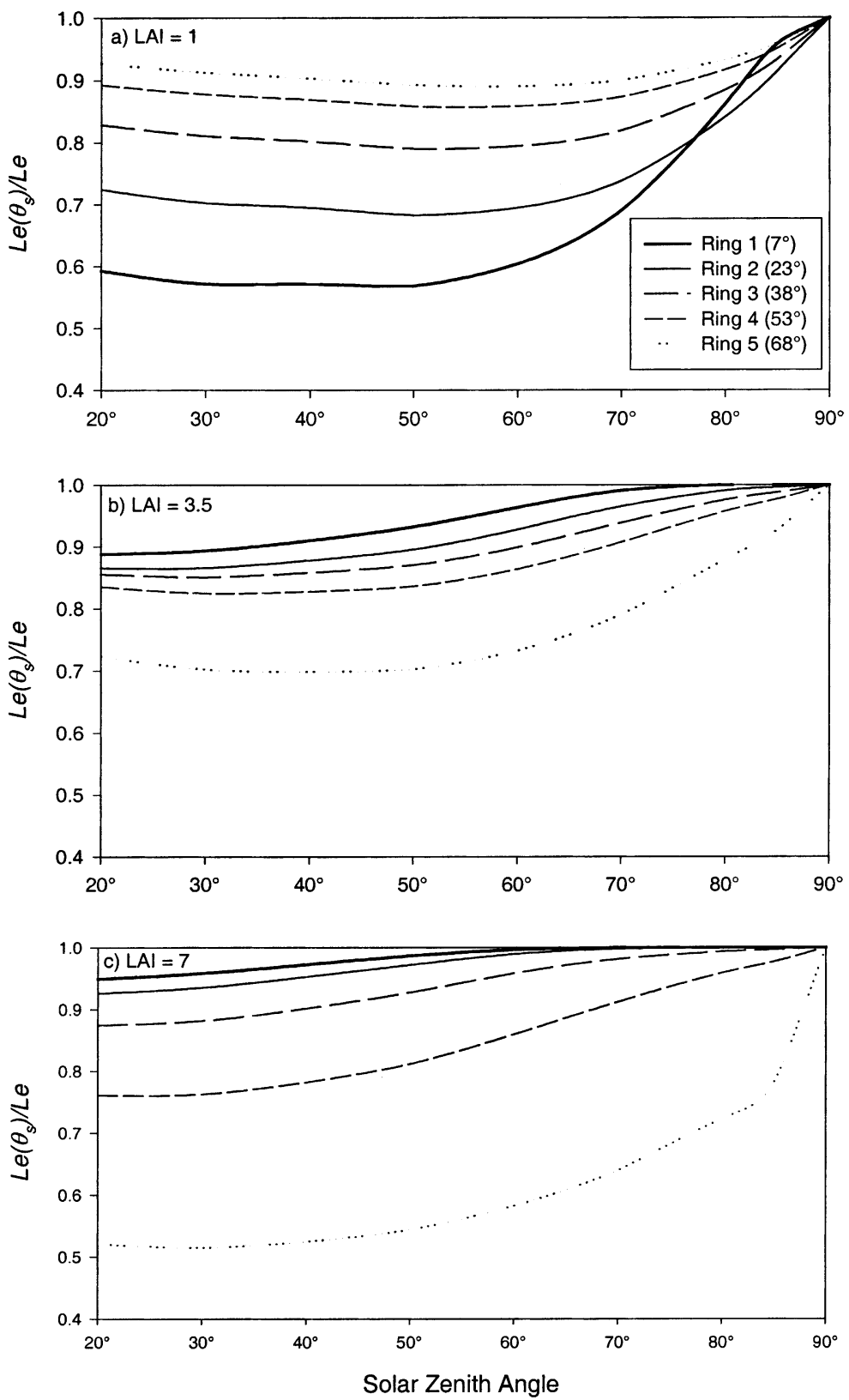

Fig. 5. Simulations of the ratio of effective LAI retrieved at solar zenith angle $\theta_{\mathrm{s}}$ to the effective LAI $\left(L_{\mathrm{e}}\left(\theta_{\mathrm{s}}\right) / L_{\mathrm{e}}\right)$ with the Five-scale radiative transfer model.

that modifies Eq. (6), has been developed to reduce the effective LAI influence

$$
\frac{L_{\mathrm{e}}\left(\theta_{\mathrm{s}}\right)}{L_{\mathrm{e}}}=A+B \mathrm{e}^{C \theta_{\mathrm{s}}}-f\left(L_{\mathrm{e}}, \theta_{\mathrm{s}}\right),
$$

where

$f\left(L_{\mathrm{e}}, \theta_{\mathrm{s}}\right)=\left[D+E L_{\mathrm{e}}\left(\theta_{\mathrm{s}}\right)\right] \frac{62-\theta_{\mathrm{s}}}{\left(90+\theta_{\mathrm{s}}\right)^{2}}$,

and $D$ and $E$ are coefficients found through fitting the 

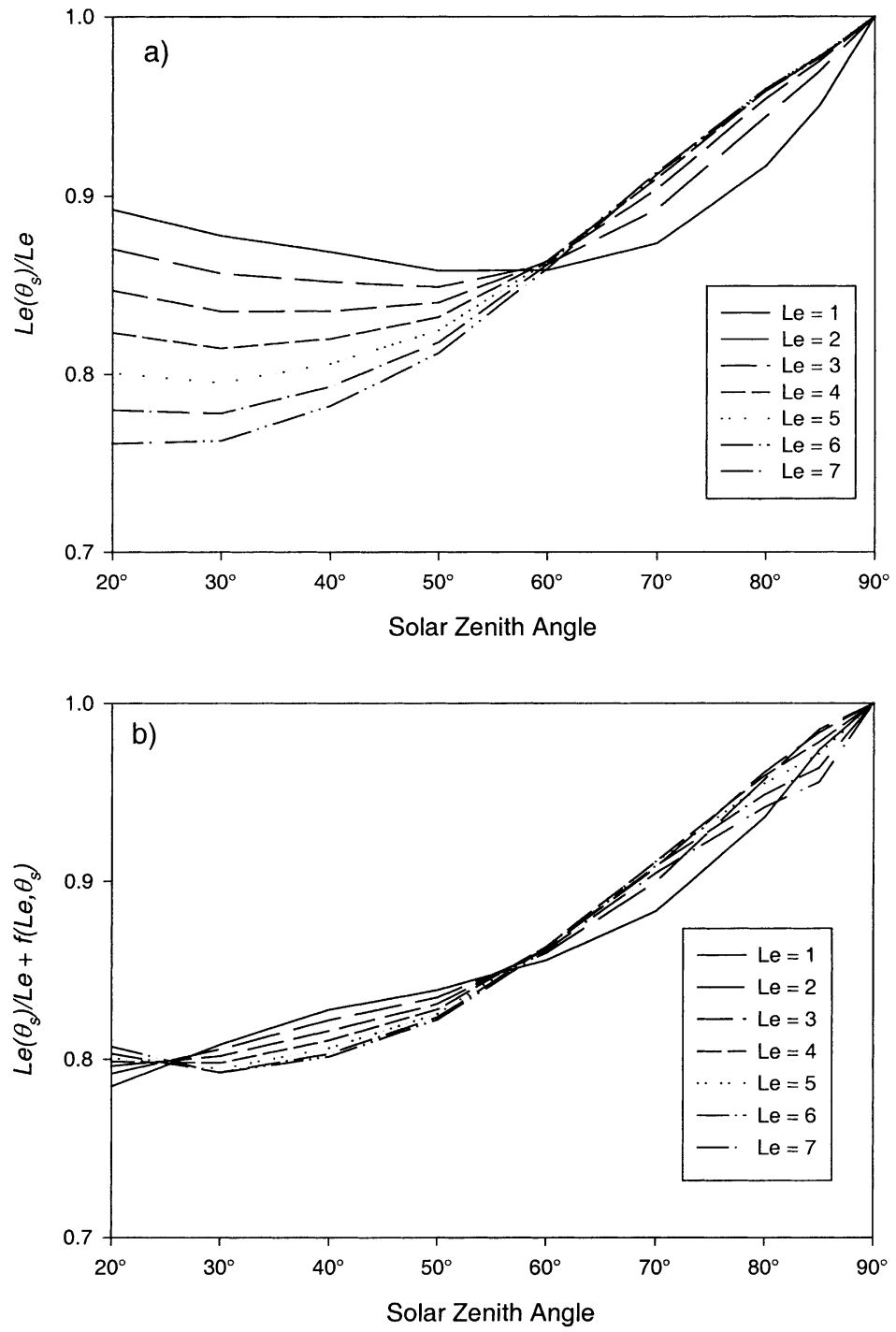

Fig. 6. Five-scale simulations of (a) the ratio of effective LAI from the LAI-2000 fourth ring retrieved at solar zenith angle $\theta_{\mathrm{s}}$ to the effective LAI $\left(L_{\mathrm{e}}\left(\theta_{\mathrm{s}}\right) / L_{\mathrm{e}}\right)$ and (b) the ratio minus the constraining function $f\left(L_{\mathrm{e}}, \theta_{\mathrm{s}}\right)$ from Eq. (10) $\left(L_{\mathrm{e}}\left(\theta_{\mathrm{s}}\right) / L_{\mathrm{e}}+f\left(L_{\mathrm{e}}, \theta_{\mathrm{s}}\right)\right)$.

model simulations. Fig. $6 \mathrm{~b}$ shows the ratio $L_{\mathrm{e}}\left(\theta_{\mathrm{s}}\right) / L_{\mathrm{e}}$ plus the additional term $f\left(L_{\mathrm{e}}, \theta_{\mathrm{s}}\right)$ as affected by $\theta_{\mathrm{s}}$ with $D=-40$ and $E=10$ found from fitting Eq. (9) to the simulation in Fig. 6a. The lines have converged together, indicating the effectiveness of Eq. (9) in removing the effects of both $\theta_{\mathrm{s}}$ and the apparent $L_{\mathrm{e}}$ on the measurements on the true $L_{\mathrm{e}}$. This is even more pronounced for angles larger than $60^{\circ}$. Values of $A=$ $0.7536, B=0.0206$ and $C=0.0278$ were found with
$R^{2}=0.987$. Inversion of Eq. (9) can then be used, knowing the solar zenith angle and $L_{\mathrm{e}}\left(\theta_{\mathrm{s}}\right)$, to retrieve $L_{\mathrm{e}}$

$$
\begin{gathered}
L_{\mathrm{e}}=\frac{L_{\mathrm{e}}\left(\theta_{\mathrm{s}}\right)}{A+B \mathrm{e}^{C \theta_{\mathrm{s}}}-\left(D+E L_{\mathrm{e}}\left(\theta_{\mathrm{s}}\right)\right)} . \\
\times\left(\left(62-\theta_{\mathrm{s}}\right) /\left(90+\theta_{\mathrm{s}}\right)^{2}\right)
\end{gathered}
$$

Based on the model-simulated dataset shown in Fig. 6a, Eq. (11) is able to retrieve $L_{\mathrm{e}}$ with errors 
smaller than $2 \%$ at all $\theta_{\mathrm{s}}$ 's. When Eq. (11) is applied to the available LAI-2000 data collected at the deciduous and conifer sites, the resulting r.m.s. difference was found to be $3 \%$ compared to a difference of $4 \%$ when using the empirical fit with Eq. (6) and $10 \%$ if the data collected under sunlit conditions are compared with the maximum $L_{\mathrm{e}}$ for that site. One precaution for applying this correction scheme, which is based on physical simulations but validated using limited empirical data, is the slight dependence of multiple scattering on the leaf reflectivity and hence on species. According to the Five-scale model, if the effective reflectivity of the foliage is $4 \%$ rather than $2 \%$ used in developing the correction scheme, the overall relative r.m.s. difference between the corrected and simulated data will increase to $12.5 \%$ for solar zenith angles covering $20-80^{\circ}$ and LAI from 1 to 7 . If the goal is to correct data taken just before the ideal diffuse light condition near sunset (using only from $\theta_{\mathrm{s}}=60$ to $90^{\circ}$ ), then doubling the reflectivity will induce a relative r.m.s. difference of only $3.5 \%$. This is still much less than the errors found without corrections, since based on the simulations, the mean relative error over all angles and LAI without correction is 13 and $21 \%$ for a foliage reflectivity of 2 and $4 \%$, respectively. When the correction scheme is applied to measurements at other wavelengths in visible range using other instruments, the error is expected to fall within the range of doubling the foliage reflectivity.

Having proved that the fourth ring is better for the sunlit day usage of the LAI-2000, it is postulated that the fourth ring can also be used alone for $L_{\mathrm{e}}$ measurements under diffuse light. A regression between the $L_{\mathrm{e}}$ from the fourth ring and all rings is shown in Fig. 7 for all data taken under diffused conditions in this paper. The relative change (slope) is about $5 \%$ between the $L_{\mathrm{e}}$ of the five rings with equal angle range $(\mathrm{d} \theta)$ and $13 \%$ between the method used by the $\mathrm{C} 2000$ software and the fourth ring. This result also holds for the $\mathrm{C} 2000$ calculation versus the fourth ring for the sunlit condition measurements. Using more than 200 plots data taken during the Canadian LAI validation (Chen et al., 2002) reveals a consistent behaviour. The fourth ring also generally agrees very well with TRAC $L_{\mathrm{e}}$ measurements at comparable view zenith angles through the canopy. Values of $L_{\mathrm{e}}$ from TRAC and from LAI-2000 are found in Table 3.

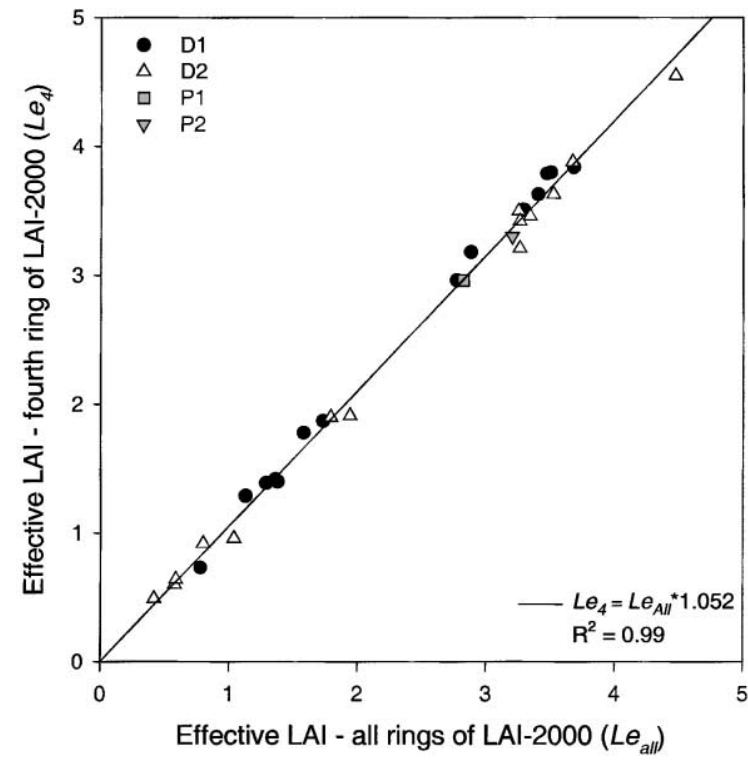

Fig. 7. Comparison between the effective LAI from LAI-2000 retrieved from all rings $\left(L_{\mathrm{e}, \text { all }}\right)$ and from ring four $\left(L_{\mathrm{e}, 4}\right)$.

\section{Conclusions}

Acquisition of plant canopy LAI with optical instruments requires optimal illumination conditions. Because of weather and time constraints, LAI field measurements are not always taken under the perfect conditions. A methodology to overcome blue light scattering problems associated with the LAI-2000 measurements taken under sunny conditions was presented. The effectiveness of the correction methodology is demonstrated through the considerable reduction of the variations in the measured LAI values of the same stands during the peak-growing season. The fourth ring of the LAI-2000 centred at the zenith angle of $53^{\circ}$ was found to be most stable for our study sites under various sunny conditions and, is therefore, suggested to be best ring for making the correction. The fourth ring also showed that the effective LAI measured under diffuse light conditions is systematically larger by $13 \%$ of the $L_{\mathrm{e}}$ values calculated with all five rings using the $\mathrm{C} 2000$ software. This value is similar to Chen et al. (1997) finding that the LAI-2000 underestimates the effective LAI due to multiple scattering by about $15 \%$. Thus using the fourth ring may be more appropriate for all conditions. The universality of the proposed correction scheme was explored using 
a canopy radiative transfer model. It appears that the scheme can be applied to stands with a wider range of LAI (1-7) than that investigated with the existing field data. The correction based on Five-scale was a small improvement over the empirical correction found from the data collected in Larose forest. The same correction scheme can be used for other instruments since the light scattering effect on LAI measurements using optical methods is an unresolved but prevailing problem.

\section{Acknowledgements}

The authors would like to thank Mr. Kevin Butler and Dr. Leonard Brown for their help in part of the fieldwork.

\section{References}

Badhwar, G.D., Macdonald, R.B., Mehta, N.C., 1986. Satellite-derived leaf-area-index and vegetation maps as input to global carbon cycle models-a hierarchical approach. Int. J. Remote Sens. 7, 265-281.

Chen, J.M., 1996. Optically-based methods for measuring seasonal variation in leaf area index of boreal conifer forests. Agric. For. Meteorol. 80, 135-163.

Chen, J.M., Black, T.A., 1991. Measuring leaf area index of plant canopies with branch architecture. Agric. For. Meteorol. 57, $1-12$.

Chen, J.M., Black, T.A., 1992. Defining leaf area index for non-flat leaves. Plant Cell Environ. 15, 421-429.

Chen, J.M., Cihlar, J., 1995. Plant canopy gap-size analysis theory for improving optical measurements of leaf area index. Appl. Opt. 34 (27), 6211-6222.

Chen, J.M., Leblanc, S.G., 1997. A four-scale bidirectional reflectance model based on canopy architecture. IEEE Trans. Geosci. Remote Sens. 35, 1316-1337.

Chen, J.M., Leblanc, S.G., 2001. Multiple scattering scheme useful for geometric optical modeling. IEEE Trans. Geosci. Remote Sens. 39, 1061-1071.

Chen, J.M., Rich, P.M., Gower, S.T., Norman, J.M., Plummer, S., 1997. Leaf area index of boreal forests: theory, techniques, and measurements. J. Geophys. Res. 102 (D24), 29429-29443.

Chen, J.M., Pavlic, G., Brown, L., Cihlar, J., Leblanc, S.G., White, H.P., Hall, R.J., Peddle, D., King, D.J., Trofymow, J.A., Swift, E., Van der Sanden, J., Pellikka, P., 2002. Validation of Canada-wide leaf area index maps using ground measurements and high and moderate resolution satellite imagery, Remote Sens. Environ., in press.

Gower, S.T., Norman, J.M., 1991. Rapid estimation of leaf area index in conifer and broad-leaf plantations. Ecology 72, 18961900.

Gower, S.T., Kucharik, C.J., Norman, J.M., 1999. Direct and indirect estimation of leaf area index, FAPAR, and net primary production of terrestrial ecosystems. Remote Sens. Environ. 70, 29-51.

Kucharik, C.J., Norman, J.M., Murdock, L.M., Gower, S.T., 1997. Characterising canopy nonrandomness with a multiband vegetation imager (MVI). J. Geophys. Res. 102 (D24), 2945529473.

Kucharik, C.J., Norman, J.M., Gower, S.T., 1998. Characterization of radiation regimes in nonrandom forest canopies: theory, measurements, and a simplified modeling approach. Tree Physiol. 19, 695-706.

Küßner, R., Mosandl, M., 2000. Comparison between direct and indirect estimation of leaf area index in mature Norway spruce stands of eastern Germany. Can. J. For. Res. 30, 440-447.

Law, B.E., Van Tuyl, A., Cescatti, A., Baldocchi, D.D., 2001. Estimation of leaf area index in open-canopy ponderosa pine forests at different successional stages and management regimes in Oregon. Agric. For. Meteorol. 108, 1-14.

Leblanc, S.G., Chen, J.M., 2000. A graphic user interface (GUI) for the Five-scale model for fast BRDF simulations. Remote Sens. Rev. 19, 293-305.

Leblanc, S.G., Chen, J.M., Kwong, M., 2001. Manual for TRAC, Ver. 2.0. Canada Centre for Remote Sensing, Ottawa, Ont.

LI-COR, 1991. LAI-2000 Plant Canopy Analyser Operating Manual. LI-COR Inc., Lincoln, NE, 90 pp.

Liu, J., Chen, J.M., Cihlar, J., Park, W.M., 1997. A process-based boreal ecosystem productivity simulator using remote sensing inputs. Remote Sens. Environ. 62, 158-175.

Miller, J.B., 1967. A formula for average foliage density. Aust. J. Bot. 15, 141-144.

Nilson, T., 1971. A theoretical analysis of the frequency of gaps in plant stands. Agric. For. Meteorol. 8, 25-38.

Privette, J.L., Myneni, R.B., Knyazikhin, Y., Mukufute, M., Roberts, G., Tian Y., Wang, Y., Leblanc, S.G., 2002. Early spatial and temporal validation of MODIS LAI product in Africa. Remote Sens. Environ. (MODIS special issue), in press.

Reid, R.R., 1979. Larose Forest. Ministry of Natural Resources, Ontario Government, Ontario, Canada.

Ross, J., 1981., The Radiation Regime and Architecture of Plant Stands. Junk, London, 391 pp.

Rudorff, B.F.T., Mulchi, C.L., Daughtry, C.S.T., Lee, E.H., 1996. Growth, radiation use efficiency, and canopy reflectance of wheat and corn grown under elevated ozone and carbon dioxide atmospheres. Remote Sens. Environ. 55, 163-173.

Smolander, H., Stenberg, P., 1996. Response of LAI-2000 estimates to changes in plant area index in a Scots pine stand. Tree Physiol. 16, 345-349.

Stenberg, P., Linder, S., Smolander, H., Flower-Ellis, J., 1994. Performance of the LAI-2000 plant canopy analyzer in estimating leaf area index of some Scots pine stands. Tree Physiol. 14, 981-995.

Thevathasan, N.V., Reynolds, P.E., Kuessner, R., Bell, W.F., 2000. Effects of controlled weed densities and soil types on soil nitrate accumulation, spruce growth, and weed growth. For. Ecol. Manage. 133, 135-144.

Warren-Wilson, J., 1965. Stand structure and light penetration. I. Analysis by point quadrats. J. Appl. Ecol. 2, 383-390.

Welles, J.M., Norman, J.M., 1991. Instrument for independent measurements of canopy architecture. Agron. J. 83, 813-825. 